\title{
Low-Noise Operation of All-Fiber Femtosecond Cherenkov Laser
}

Liu, Xiaomin ; Villanueva Ibáñez, Guillermo Eduardo; Lægsgaard, Jesper; Møller, Uffe Visbech; Tu, Haohua; Boppart, Stephen A.; Turchinovich, Dmitry

Published in:

I E E E Photonics Technology Letters

Link to article, DOI:

10.1109/LPT.2013.2253765

Publication date:

2013

Link back to DTU Orbit

Citation $(A P A)$ :

Liu, X., Villanueva Ibáñez, G. E., Lægsgaard, J., Møller, U. V., Tu, H., Boppart, S. A., \& Turchinovich, D. (2013). Low-Noise Operation of All-Fiber Femtosecond Cherenkov Laser. I E E E Photonics Technology Letters, 25(9), 892-895. https://doi.org/10.1109/LPT.2013.2253765

\section{General rights}

Copyright and moral rights for the publications made accessible in the public portal are retained by the authors and/or other copyright owners and it is a condition of accessing publications that users recognise and abide by the legal requirements associated with these rights.

- Users may download and print one copy of any publication from the public portal for the purpose of private study or research.

- You may not further distribute the material or use it for any profit-making activity or commercial gain

- You may freely distribute the URL identifying the publication in the public portal

If you believe that this document breaches copyright please contact us providing details, and we will remove access to the work immediately and investigate your claim 


\title{
Low-noise operation of all-fiber femtosecond Cherenkov laser
}

\author{
Xiaomin Liu, Guillermo E. Villanueva, Jesper Lægsgaard, Uffe Møller, Haohua Tu, Stephen A. Boppart, and \\ Dmitry Turchinovich
}

\begin{abstract}
We investigate the noise properties of a femtosecond all-fiber Cherenkov radiation source with emission wavelength around $600 \mathrm{~nm}$, based on an Yb-fiber laser and a highly-nonlinear photonic crystal fiber. A relative intensity noise as low as $103 \mathrm{dBc} / \mathrm{Hz}$, corresponding to $2.48 \%$ pulse-to-pulse fluctuation in energy, was observed at the Cherenkov radiation output power of $4.3 \mathrm{~mW}$, or $150 \mathrm{pJ}$ pulse energy. This pulse-to-pulse fluctuation is at least $10.6 \mathrm{~dB}$ lower compared to spectrally-sliced supercontinuum sources traditionally used for ultrafast fiberbased generation at visible wavelengths. Low noise makes allfiber Cherenkov sources promising for biophotonics applications such as multi-photon microscopy, where minimum pulse-to-pulse energy fluctuation is required. We present the dependency of the noise figure on both the Cherenkov radiation output power and its spectrum.
\end{abstract}

Index Terms-Ultrafast optics, fiber lasers, laser noise, noise measurement.

\section{INTRODUCTION}

$\mathbf{F}$ IBER-OPTIC Cherenkov radiation (CR), also known as dispersive wave generation or non-solitonic radiation, is produced in small-core photonic crystal fibers (PCF) when a soliton perturbed by fiber higher-order dispersion copropagates with a dispersive wave fulfilling a certain phasematching condition [1], [2].

The rapid development of PCF and ultrafast laser technologies has led to significant interest in resonant ultrafast wave conversion via the fiber-optic CR mechanism [3], [4], [5], [6], [7], in particular with applications in biophotonics, such as bio-imaging and microscopy [8]. The sensitivity of bio-imaging and microscopy systems, one of the key parameters for the system performance evaluation, is typically limited by the intensity noise of the light source used in the system [9]. Further, practical biophotonics applications require light sources with high long-term operational stability, which makes the all-fiber systems not containing any free

X. Liu, J. Lægsgaard and U. Møller are with DTU Fotonik, Technical University of Denmark, DK-2800 Kgs. Lyngby, Denmark (e-mail: xali@fotonik.dtu.dk; jlag@ fotonik.dtu.dk; ufmo@ fotonik.dtu.dk).

Guillermo E. Villanueva is with DTU Fotonik, Technical University of Denmark, DK-2800 Kgs. Lyngby, Denmark and Nanophotonics Technology Center, Universitat Politècnica de València, 46022 Valencia, Spain (e-mail: guivilib@teleco.upv.es).

$\mathrm{H}$. Tu, and S. A. Boppart are with Biophotonics Imaging Laboratory, University of Illinois at Urbana-Champaign, Urbana, Illinois 61801, U.S.A. (e-mail: htu@illinois.edu; boppart@illinois.edu)

D. Turchinovich is with DTU Fotonik, Technical University of Denmark, DK-2800 Kgs. Lyngby, Denmark and Max Planck Institute for Polymer Research, Ackermannweg 10, 55128 Mainz, Germany (e-mail: dmtu@fotonik.dtu.dk)

Manuscript received January 19, 2013

Copyright (c) 2012 IEEE. space components the natural choice [10]. CR, producing the spectrally-isolated output at an arbitrary wavelength, is a new alternative to spectrally-sliced supercontinuum, traditionally generated in highly-nonlinear PCFs pumped by near-tansformlimited picosecond pulses (ps-SC). The unfiltered CR with the highest power can be directly used in real-life bio-imaging applications. For example, with a spectral coverage of 540$680 \mathrm{~nm}$, it is an excellent source in two-photon fluorescence microscopy to simultaneously stimulate multiple biomolecules such as tryptophan, tyrosine, NADH, flavin, and collagen. The different emission bands from these biomolecules allow for spectrally resolved detection to differentiate between different biomolecules. Filtered CR is also useful because different biomolecules may be selectively excited.

Recently, we have succeeded in demonstration of an efficient Cherenkov femtosecond laser emitting around $600 \mathrm{~nm}$, based on an all-fiber architecture and featuring extremely-high mechanical and operational stability [7]. Since both the CR lasers and the ps-SC sources are mainly intended for use in practical biophotonics, it is especially important to compare the noise properties of both types of systems.

In this Letter we report on the experimental characterization of intensity noise of an all-fiber Cherenkov laser, to the best of our knowledge, for the first time. We demonstrate that the relative intensity noise (RIN) of the CR generated around $600 \mathrm{~nm}$ wavelength is as low as $-103 \mathrm{dBc} / \mathrm{Hz}$, corresponding to pulse-to-pulse energy fluctuation of $2.48 \%$. As will be shown below, this energy fluctuation value is about $10.6 \mathrm{~dB}$ lower than that of an all-fiber ps-SC source spectrally-sliced to produce the output at the same central wavelength.

\section{SYSTEM DESCRIPTION}

Our home-built all-fiber femtosecond CR source [7] consists of two main parts: a monolithic Yb-fiber nJ-level selfstabilized [11] femtosecond master oscillator - power amplifier (MOPA) [12] operating at a wavelength of $1035 \mathrm{~nm}$ and a repetition rate of $26.7 \mathrm{MHz}$, used as the pump source; and a spliced-on small-core nonlinear PCF NL-3.0-850 (NKT Photonics A/S) with zero-dispersion wavelength around 850 nm, used for Cherenkov wave conversion. Bridge fibers are used in the CR link to increase the incoupling efficiency. Our CR source features no free-space optical coupling: all its fibers are spliced together. It produces electrically wavelengthtunable CR output in the spectral range of $580-630 \mathrm{~nm}$, with average power up to $5 \mathrm{~mW}$, and with sub-160 fs pulse duration. The typical pulse autocorrelation and far field saturated visible 

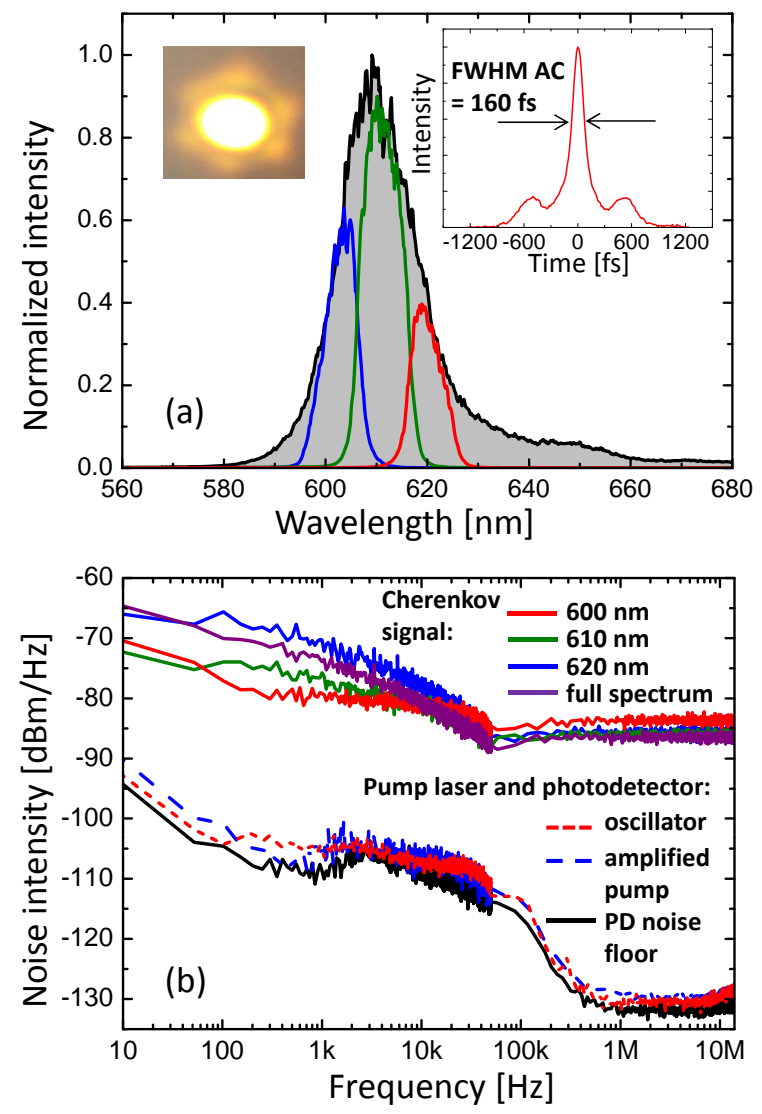

Fig. 1. (a) CR spectrum with and without band-pass optical filters. The power of un-filtered CR is $0.54 \mathrm{~mW}$. Inset: autocorrelation (right) and far field saturated visible image (left) of typical CR output; (b) Noise spectra of: CR measured with and without bandpass optical filters with the same detected optical power (solid lines); MOPA oscillator (red dash-dotted line); full MOPA (blue dashed line). Noise floor of the photoreceiver (black line).

image of the CR output are shown in the inset of Fig. 1(a). See Ref. [7] and references therein for more details on this system.

The intensity noise of our CR system was characterized as described in Refs. [13], [14]. The CR signal was first filtered out from the pump signal and then detected with a photoreceiver (PR) (New Focus, Inc. $125 \mathrm{MHz}$ ), which was connected to an electrical spectrum analyzer (ESA) (Agilent EXA N9010A) to measure the noise spectrum, and to a voltmeter to measure the average optical power. For spectrallydependent intensity noise measurements, the bandpass optical filters with $10 \mathrm{~nm}$ FWHM bandwidth, and with central wavelength ranging from $570 \mathrm{~nm}$ to $620 \mathrm{~nm}$, were inserted individually into a beampath leading to the PR.

\section{EXPERIMENTAL RESULTS AND ANALYSIS}

Fig. 1(a) shows the optical spectrum of the typical CR output from our system as measured with and without the bandpass optical filters. The power of un-filtered CR in this case was $0.54 \mathrm{~mW}$. The far field saturated visible image of typical CR output is shown in the left inset of Fig. 1(a). The CR output is generated in the fundamental mode with a symmetric mode profile very similar to Gaussian profile (see e.g. [10] for a typical PCF-laser generated mode), so that minimum spatial degradation is expected in bioimaging applications. The noise spectra of the filtered and un-filtered CR signals were measured in the baseband of the electrical frequency ranging from $10 \mathrm{~Hz}$ to $13.35 \mathrm{MHz}$ (half of the repetition rate of the pump laser source) with the same detected optical power, and are shown in Fig. 1(b). In Fig. 1(b), the black solid line shows the noise floor of the PR; the red dash-dotted line indicates the noise spectrum of the MOPA oscillator alone [11], while the blue dashed line shows the noise spectrum of the full pump MOPA, as measured before launching its output into the CR generation fiber link. Note that the noise levels of both the oscillator and the full MOPA, measured at any output power, are virtually indistinguishable from the PR noise level, i.e. the real noise level of the pump laser is too low to be resolved by our PR.

The four solid lines in the upper part of Fig. 1(b) show the noise power of the CR output from Fig. 1(a) with and without optical bandpass filtering. Since the noise power of CR is at least $20 \mathrm{~dB}$ higher than the noise floor of PR as shown in Fig. 1(b), the noise properties of the CR can be accurately quantified in terms of relative intensity noise (RIN) as a function of electrical frequency and input power [14]. Due to the limitation of the bandwidth resolution (BW) and sweeping time of our ESA, we used different BW for different frequency ranges: $1 \mathrm{~Hz} \mathrm{BW}$ was used for the noise measurements in the range $10 \mathrm{~Hz}-50 \mathrm{kHz}$, and $1 \mathrm{kHz} \mathrm{BW}$ for the range $50 \mathrm{kHz}$ 13.35 MHz, producing the change of the noise spectra at the border frequency of $50 \mathrm{kHz}$.

The noise in the CR generation process can be divided into two contributions: a low-frequency part originating from technical laser noise and a broadband frequency part originating from amplified quantum noise [15], [16]. The lowfrequency component of the intensity noise results mainly from the amplification of the input noise of the pump laser. The broadband frequency component of the intensity noise at the electrical frequency above $1 \mathrm{MHz}$ in Fig. 1(b) arises from the two fundamental quantum noise seeds: the input shot noise, and spontaneous Raman scattering along the PCF fiber [16]. RIN, which describes the intensity noise properties, is quantified by the noise power in a $1 \mathrm{~Hz}$ bandwidth normalized to the DC signal power [13]. The pulse-to-pulse energy fluctuations can be calculated as a square root of RIN integrated in frequency $f$ from a certain frequency $f_{0}$ and up to half the repetition rate $f_{N}=$ rep. rate/2 [17]. The signal to noise ratio (SNR) is defined as the reciprocal of the pulse-topulse energy fluctuations, SNR $=1 / \sqrt{\int_{f_{0}}^{f_{N}} \text { RIN } d f}$. We note that for primary applications of CR sources such as nonlinear microscopy, the low-frequency noise part can be removed by high-frequency modulation of the excitation beam along with lock-in detection of the signal. In this arrangement only the broadband frequency noise part contributes to the pulse-topulse fluctuations, and therefore $f_{0}=1 \mathrm{MHz}$ was chosen for SNR calculation in this work. Fig. 2(a) shows the RIN of CR without optical bandpass filtering, dependent on the average input pump power at $1035 \mathrm{~nm}$, and output CR power. With the shot noise limit of the measurement system around $-130 \mathrm{dBc} / \mathrm{Hz}$, the broadband, high-frequency RIN decreases 

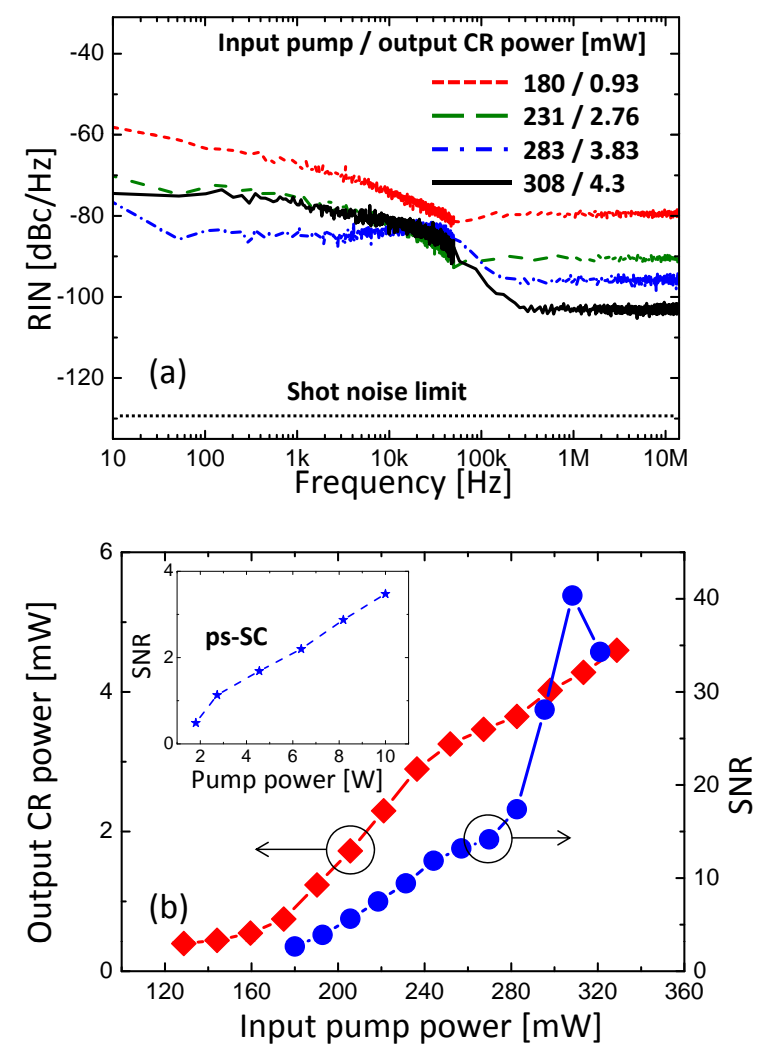

Fig. 2. Measurements on spectrally-unfiltered CR. (a) RIN of CR generated at different input pump powers; shot noise limit of the measurement (dotted line); (b) SNR measured from $1 \mathrm{MHz}$ to $13.35 \mathrm{MHz}$ (blue circles), and output power (red diamonds) of CR dependent on the input pump power. (Inset) SNR from $1 \mathrm{MHz}$ to $40 \mathrm{MHz}$ (blue stars) of the output of a typical all-fiber ps-SC source, spectrally sliced at $600 \mathrm{~nm}$, as a function of the input pump power.

dramatically from $-80 \mathrm{dBc} / \mathrm{Hz}$ to $-103 \mathrm{dBc} / \mathrm{Hz}$, when the average input pump power increases from $180 \mathrm{~mW}$ to $308 \mathrm{~mW}$, and the output CR power grows from $0.93 \mathrm{~mW}$ to $4.3 \mathrm{~mW}$. Fig. 2(b) summarizes the dependency of the average power and SNR of optically-unfiltered CR on the input pump power at $1035 \mathrm{~nm}$. When the input pump is increased to $308 \mathrm{~mW}$, the SNR of CR reaches the value of 40.4 , corresponding to a pulse energy fluctuation as low as $2.48 \%$ with the average output power of $4.3 \mathrm{~mW}$.

For comparison, in the inset of Fig. 2(b) we show the SNR of the output of a standard all-fiber ps-SC source based on an Yb-fiber pump MOPA with repetition of $80 \mathrm{MHz}$, spectrally sliced with $10 \mathrm{~nm}$ bandwidth (FWHM) optical bandpass filter around the wavelength of $600 \mathrm{~nm}$, as a function of the input pump power [18], [19]. The ps-SC source shows highest SNR of 3.48 , corresponding to pulse energy fluctuations of 28.78 $\%$, which is $10.6 \mathrm{~dB}$ larger than that of our all-fiber CR source operating around the same wavelength of $600 \mathrm{~nm}$. Note the much higher pump power of a ps-SC source reaching $10 \mathrm{~W}$, as compared to sub-Watt pumping of an all-fiber CR source. The $10.6 \mathrm{~dB}$ difference in SNR between the CR and ps-SC sources means that, owing to the square-root law of error propagation in signal averaging, it will take about 130 individual measurements for the ps-SC source to achieve the same quality of data, as only one measurement using the
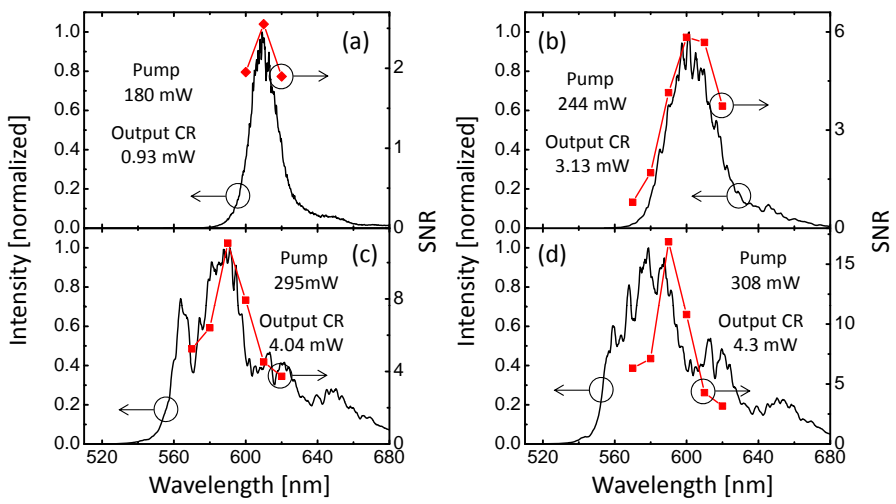

Fig. 3. Spectra of CR output (black line) and of SNR measured at different spectral positions (red diamond) for different MOPA pump and CR output powers.

$\mathrm{CR}$ source. A later measurement on another ps-SC source indicated an SNR increase of $2.2 \mathrm{~dB}$ when the bandwidth of the bandpass filter increases from $10 \mathrm{~nm}$ to $40 \mathrm{~nm}$.

Finally, we demonstrate the spectral distribution of SNR as a function of the CR output power. For different MOPA pump and output CR powers, Fig. 3 shows the optical intensity spectra of the spectrally-unfiltered CR, and the SNR spectra measured from the bandpass-filtered CR signals such as shown in Fig. 1(a). In general agreement with the data presented in Fig. 2(a), the SNR increases with increase in CR output power. At the CR average power of $3.2 \mathrm{~mW}$ and lower, the SNR spectrum coincides with the optical intensity spectrum, as shown in Fig. 3(a) and (b), indicating the constant absolute noise level within the $\mathrm{CR}$ spectrum. However, when the average $\mathrm{CR}$ power is higher than $4 \mathrm{~mW}$, the SNR increases to the highest values at the CR central wavelength, while decreasing rapidly away from the CR central wavelength as shown in Fig. 3(c) and (d). Further, we found that the highest SNR value in the case of spectrally filtered CR is considerably lower than the peak value of 40.4 observed for the unfiltered output. Our preliminary numerical simulations indicate a significant spectral-domain jitter of the Cherenkov signal, which may explain the observed trends of decreasing SNR towards the spectral edges, as well as lower peak SNR for the bandwidth-filtered CR as compared to SNR measured for the whole spectrum (Fig. 2(b)). This phenomenon is subject to further investigation.

\section{CONCLUSION}

In summary, we have experimentally investigated the intensity noise of an all-fiber femtosecond CR source with electrical wavelength tunability in the range $580 \mathrm{~nm}-630 \mathrm{~nm}$, and multimilliwatt output powers. When the output average power reaches $4.3 \mathrm{~mW}$, a spectrally-averaged RIN as low as -103 $\mathrm{dBc} / \mathrm{Hz}$ is measured, corresponding pulse energy fluctuation as low as $2.48 \%$, which is $10.6 \mathrm{~dB}$ lower than that of an all-fiber ps-SC source pumped with much higher power. This low noise level of the all-fiber CR source makes it promising for practical biophotonics applications such as e.g. scanning fluorescence microscopy, where the low pulse-to-pulse energy 
fluctuations is a requirement. At higher CR output a nonlinear dependency of SNR spectral distribution on the CR power is observed, which may be caused by significant spectral-domain jitter in Cherenkov output. Further investigations are needed to clarify this point.

\section{ACKNOWLEDGMENT}

The authors would like to thank the Danish Council for Independent Research - Technology and Production Sciences (FTP), and the National Institutes of Health (grants R01 EB012479 and R01 CA166309) for financial support; A. S. Olesen and T. Mikelsen for valuable assistance; and NKT Photonics A/S for providing the PCFs.

\section{REFERENCES}

[1] P. K. A. Wai, C. R. Menyuk, Y. C. Lee, and H. H. Chen, "Nonlinear pulse propagation in the neighborhood of the zero-dispersion wavelength of monomode optical fibers," Opt. Lett., vol. 11, no. 7, pp. 464-466, Jul. 1986.

[2] N. Akhmediev and M. Karlsson, "Cherenkov radiation emitted by solitons in optical fibers," Phy. Rev. A, vol. 51, no. 3, pp. 2602-2607, Mar. 1995.

[3] F. Lu and W. H. Knox, "Generation, characterization, and application of broadband coherent femtosecond visible pulses in dispersion micromanaged holey fibers," J. Opt. Soc. Am. B, vol. 23, no. 6, pp. 1221-1227, Jun. 2006.

[4] H. Tu and S. A. Boppart, "Optical frequency up-conversion by supercontinuum-free widely-tunable fiber-optic Cherenkov radiation," Opt. Express, vol. 17, no. 12, pp. 9858-9872, Jun. 2009.

[5] G. Chang, L. J. Chen, and F. X. Kärtner, "Highly efficient Cherenkov radiation in photonic crystal fibers for broadband visible wavelength generation," Opt. Lett., vol. 35, no. 14, pp. 2361-2363, Jul. 2010.

[6] A. Choudhary and F. König, "Efficient frequency shifting of dispersive waves at solitons," Opt. Express, vol. 20, no. 5, pp. 5538-5546, Feb. 2012.

[7] X. Liu, J. Lægsgaard, U. Møller, H. Tu, S. A. Boppart, and D. Turchinovich, "All-fiber femtosecond Cherenkov radiation source," Opt. Lett., vol. 37, no. 13, pp. 2769-2771, Jul. 2012.

[8] H. Tu and S. A. Boppart, "Coherent fiber supercontinuum for biophotonics," Laser Photon. Rev., DOI: 10.1002/lpor.201200014.

[9] S. Shin, U. Sharma, H. Tu, W. Jung, and S. A. Boppart, "Characterization and analysis of relative intensity noise in broadband optical sources for optical coherence tomography," IEEE Photon. Technol. Lett., vol. 22, no. 14, pp. 1057-1059, Jul. 2010.

[10] X. Liu, J. Lægsgaard, and D. Turchinovich, "Monolithic highly-stable $\mathrm{Yb}$-doped femtosecond fiber lasers for applications in practical biophotonics," J. Sel. Top. Quant. Electron., vol. 18, no. 4, pp. 1439-1450, Jul.-Aug. 2012.

[11] X. Liu, J. Lægsgaard, and D. Turchinovich, "Self-stabilization of a mode-locked femtosecond fiber laser using a photonic bandgap fiber," Opt. Lett., vol. 35, no. 7, pp. 913-915, Apr. 2010.

[12] X. Liu, J. Lægsgaard, and D. Turchinovich, "Highly-stable monolithic femtosecond Yb-fiber laser system based on photonic crystal fibers," Opt. Express, vol. 18, no. 15, pp. 15475-15483, Jul. 2010.

[13] D. Derickson, ed., Fiber Optic Test and Measurement (Prentice Hall, 1998).

[14] R. P. Scott, C. Langrock, and B. H. Kolner, "High-dynamic-range laser amplitude and phase noise measurement techniques," J. Sel. Top. Quant. Electron., vol. 7, no. 4, pp. 641-655, Jul.-Aug. 2001.

[15] N. R. Newbury, B. R. Washburn, K. L. Corwin, and R. S. Windeler, "Noise amplification during supercontinuum generation in microstructure fiber," Opt. Lett., vol. 28, no. 11, pp. 944-946, Jun. 2003.

[16] K. L. Corwin, N. R. Newbury, J. M. Dudley, S. Coen, S. A. Diddams, K. Weber, and R. S. Windeler, "Fundamental noise limitations to supercontinuum generation in microstructure fiber," Phys. Rev. Lett., vol. 90, no. 11, pp. 113904, Mar. 2003.

[17] U. Møller, S. T. Sørensen, C. Larsen, P. M. Moselund, C. Jakobsen, J. Johansen, C. L. Thomsen, and O. Bang, "Optimum PCF tapers for blueenhanced supercontinuum sources," Opt. Fiber Technol., vol. 18, no. 5, pp. 304-314, Sep. 2012.

[18] U. Møller, S. T. Sørensen, C. Jakobsen, J. Johansen, P. M. Moselund, C. L. Thomsen, and O. Bang, "Power dependence of supercontinuum noise in uniform and tapered PCFs," Opt. Express, vol. 20, no. 3, pp. 2851-2857, Jan. 2012.
[19] U. Møller, S. T. Sørensen, C. Jakobsen, J. Johansen, P. M. Moselund, C. L. Thomsen, and O. Bang, "Power dependence of supercontinuum noise in uniform and tapered PCFs: erratum" Opt. Express, vol. 20, no. 21, pp. 23318-23319, Oct. 2012. 